\title{
Premières données sur les variations saisonnières de la communauté d'oiseaux du barrage urbain de Koko (Korhogo, Côte d'Ivoire)
}

NIAMIEN Coffi Jean Magloire ${ }^{1}$, KONAN Ekoun Michaël ${ }^{1}$, ODOUKPE Kadio Saint Guillaume $^{2}$, YAOKOKORE-BEIBRO Kouassi Hilaire ${ }^{2}$ et N'GORAN Kouakou Eliézer ${ }^{2}$

${ }^{1}$ Département de Biologie Animale, UFR des Sciences Biologiques, Université Peleforo Gon Coulibaly, BP 1328 Korhogo, Côte d'Tvoire

${ }^{2}$ Laboratoire de Zoologie et Biologie Animale, UFR Biosciences, Université Félix Houphouët-Boigny, 22 BP 582 Abidjan, Côte d'Ivoire

Auteur correspondant : niamien.coffi@gmail.com; Tel: +22557985568

Mots clés : Zone humide, Oiseaux, diversité, effet de la saison, Korhogo, Côte d'Ivoire.

Key words: Wetland, birds, diversity, season effect, Korhogo, Côte d'Ivoire.

Publication date 31/08/2019, http://www.m.elewa.org/JAPS

\section{RÉSUMÉ}

Les oiseaux rendent d'importants services écologiques nécessaires au bien-être de l'homme. En Côte d'Ivoire, l'essentiel des études ornithologiques se sont déroulées au sud, et accessoirement au centre et au centre-ouest. En outre, très peu de travaux ont porté sur les zones humides. La ville de Korhogo abrite des barrages à vocation agro-pastorale qui n'ont été l'objet d'aucune étude. Pour combler ce manque de connaissance, une étude a été menée de février 2016 à janvier 2017. L'objectif de ce travail est d'évaluer la richesse spécifique et de déterminer l'influence des saisons sur la distribution qualitative de la communauté des oiseaux du barrage urbain de Koko dans la commune de Korhogo. Pour ce faire, des observations directes, utilisant la méthode des transects ponctuée d'arrêts d'environ 15 minutes, ont été effectuées. Les résultats indiquent que cette communauté est constituée de 63 espèces d'oiseaux appartenant à 32 familles et regroupées en 14 ordres. Globalement, l'ordre des Passériformes et la famille des Ardeidae sont les mieux représentés. Du point de vue de leur statut biogéographique, les espèces résidentes (37 individus : $59 \%$ ) et accidentelles (23 individus : 36,51\%) sont majoritaires. Le plus grand nombre d'espèces a été observé en saison des pluies (52 individus : 82,54\%). Sept espèces ont été spécifiques de la saison sèche alors que trois espèces sont caractéristiques de la saison des pluies. Les espèces résidentes ont été les nombreuses en toute saison. Au niveau structural, les espèces accessoires, les espèces assez-fréquentes et les espèces fréquentes ont été les mieux représentées en saison sèche tandis que les espèces assez-fréquentes ont été majoritaires en saison des pluies. Cette étude confirme la distribution des communautés des oiseaux selon les saisons.

\section{ABSTRACT}

Birds provide important ecosystem services necessary for human well-being. In Côte d'Ivoire, ornithological studies were carried out in the south, central and central-west. In addition, very little work has been done on wetlands. The Commune of Korhogo is home to agro-pastoral dams that have not been studied. To fill in this gap of information, a study was conducted from February 2016 to January 2017. The aim of this study was to assess the 
specific richness and to examine the effect of seasons on the qualitative distribution of the Koko urban dam birds' community. Direct observations using the method of line transects with a 15 minutes points were carried out. Results show that this community consists of 63 species of birds belonging to 32 families and grouped in 14 orders. Globally, the order of the Passeriformes and the family of Ardeidae were the best represented. In terms of their biogeographical status, resident species (37 individuals: 59\%) and accidental species (23 individuals: $36.51 \%$ ) were dominant. The high number of bird species was observed during the rainy season (52 individuals: $82.54 \%$ ). Seven species were specific to the dry season while three species are characteristic of the rainy season. Resident species were numerous in all seasons. The accessory and relatively frequent species have been the most abundant during the dry season while the fairly frequent species were predominant in the rainy season. This study confirms the distribution of bird communities according to the seasons.

\section{INTRODUCTION}

Les écosystèmes naturels sont sous la pression des activités humaines avec pour corollaire la perte de la biodiversité (Khaffou et al., 2013 ; Konan et al., 2014). Pour une gestion durable des écosystèmes, la connaissance des communautés, les spécificités d'habitats, les variations d'effectifs et les menaces doivent être prises en compte (Conservation International, 2001). Pour ce faire, les données relatives aux facteurs de variations des communautés sont indispensables pour la mise en place de stratégies de conservation. Les zones humides fournissent divers services écosystémiques ainsi que de l'eau dont dépendent des organismes végétaux et animaux pour leur survie. L'homme en tire profit dans le cadre des activités agropastorales (Odoukpé et al., 2014). Par ailleurs, ces milieux de fortes disponibilités de ressources alimentaires abritent une forte diversité avifaunique (Ahmad et Bhat, 2017). En effet, ces zones humides sont des sites de nutrition, d'hivernage et de reproduction pour de nombreuses espèces d'oiseaux d'eau migratrices et résidentes dont certaines sont inscrites sur la liste rouge de l'Union Internationale de la Conservation de la Nature (El Agbani et Dakki, 2005 ; Borrow et Demey, 2008 ; Yaokokoré-Béibro, 2010). Par ailleurs, cette présence des espèces d'oiseaux est utilisée comme indicateur pour mesurer l'état de santé des écosystèmes à travers le niveau de pollution (Bryce et al., 2002 ; Ahmad et Bhat, 2017). Dès lors, ces milieux devraient être considérés comme des sites stratégiques pour la conservation de la biodiversité. Cependant, ces écosystèmes à fort potentiel écologique et de conservation sont menacés de disparition du fait des activités anthropiques, qui entrainent la perte de la diversité biologique (Williams, 1993 ; Brooks et al., 2007 ; Ahmad et Bhat, 2017). En Côte d'Ivoire, les travaux portant sur l'avifaune se sont essentiellement déroulés en milieu forestier dans la partie sud du pays et accessoirement au centre et au centre-ouest (Kouadio et al., 2014; Odoukpé et al., 2014 ; Konan et al., 2015 ; Yaokokoré-Béibro et al., 2015 a, b; Zean et al., 2018). Les études ornithologiques relatives aux zones humides sont peu nombreuses et se sont concentrées à Grand-Bassam, à Yamoussoukro et accessoirement à Abidjan (Odoukpé et al., 2014 ; Konan et al., 2015 ; Yaokokoré-Béibro et al., 2015 a, b). Le nord du pays abrite divers barrages à vocation agro-pastorale. Ces barrages n'ont pas été l'objet d'étude portant sur l'avifaune. Cette étude a été menée pour pallier ce manque d'information. Elle est une contribution à une meilleure connaissance de l'écologie des oiseaux afin d'en assurer une gestion durable et rationnelle. Elle vise spécifiquement à (i) inventorier les oiseaux du barrage urbain de Koko et à (ii) examiner l'influence des saisons sur la composition et la distribution qualitative de la communauté. 


\section{MATERIEL ET METHODES}

3.1 Site d'étude : Le département de Longitude) fait partie du District des savanes et Korhogo (9³4' de Latitude Nord et $5^{\circ} 37^{\prime}$ de de la région du Poro (Figure 1).

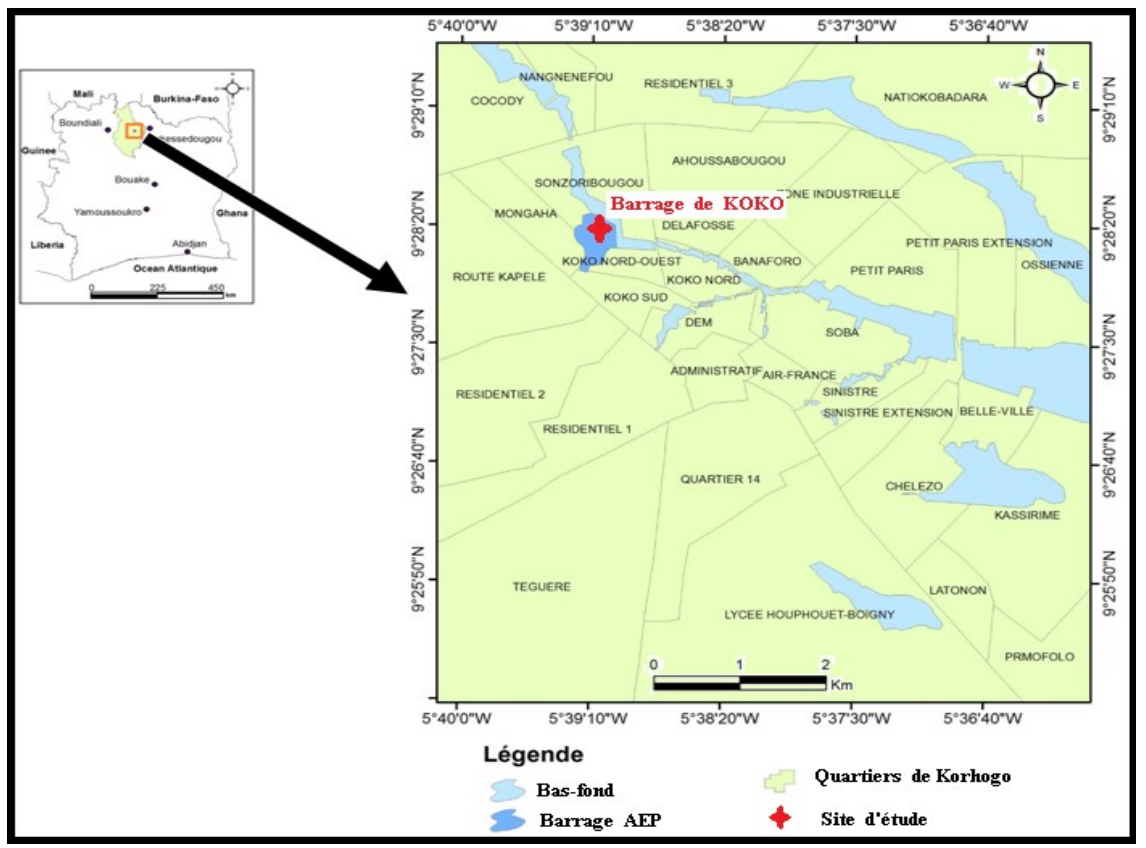

Figure 1 : Localisation de la commune de Korhogo et du site d'étude

Le climat de la ville de Korhogo est de type soudanais à deux saisons : une saison sèche (de novembre à mars) et une saison des pluies (d'avril à octobre). Les fluctuations du niveau d'eau sont fonction des saisons (Coulibaly, 2014). La ville de Korhogo abrite plusieurs barrages. Dans le quartier de Mongaha, le barrage de Koko est utilisé comme source d'approvisionnement en eau par la Société de Distribution d'Eau de Côte d'Ivoire. Par ailleurs, autour de ce barrage s'est développé des cultures céréalières (en saison des pluies) et des cultures maraichères (saison sèche).

3.2 Collecte des données : Les données relatives à l'avifaune du barrage urbain de Koko ont été collectées sur un cycle annuel de février 2016 à janvier 2017. La méthode d'observations directes utilisant des transects ponctués par des arrêts d'environ 15 minutes a été utilisée (Yaokokoré-Béibro, 2001; Odoukpé et al., 2014 ; Yaokokoré-Béibro et al., 2015 a, b). Pour ce faire, nous avons marché le long des contours du barrage afin d'identifier toutes les espèces d'oiseaux présentes sur le plan d'eau et/ou aux alentours du barrage jusqu'à 50 mètres à partir de la berge (Konan et al., 2015). Les visites ont été effectuées par beau temps, deux fois par mois. Les inventaires d'oiseaux ont été réalisés de 07 h 00 à 10 h 00 , tranche horaire, qui correspond à une période de forte activité (Bibby et al., 1992 ; Yaokokoré-Béibro, 2001 ; Yaokokoré-Béibro et al., 2015 a et b). Tous les oiseaux vus, entendus, posés ou au vol ont été observés à la jumelle et identifiés à l'aide $\mathrm{du}$ guide d'identification des oiseaux de l'Afrique de l'Ouest (Borrow et Demey, 2008). Par ailleurs, les chants et cris d'espèces inconnues, ont été enregistrés avec un dictaphone et reconnus grâce au CD-Rom des chants et cris des oiseaux d'Afrique (Chappuis, 2000). La séquence des ordres et des familles est conforme à la liste systématique de Borrow et Demey (2001). La liste des espèces observées donnent pour chacune d'elles, le statut biogéographique (Borrow et Demey, 2001) ainsi que la fréquence d'occurrence (Djakou et Thanon, 1988) calculée sur la base de leur 
présence saisonnière. L'expression de cette fréquence d'occurrence (Fo) est la suivante :

$\mathrm{Fo}_{\mathrm{O}}=(\mathrm{Si} / \mathrm{St}) \times 100$

Où : $\mathrm{Si}=$ nombre de relevés où l'espèce i a été présente,

$\mathrm{St}=$ nombre total de relevés.

Selon Djakou et Thanon (1988), une espèce est dite espèce très fréquente si $80 \%<\mathrm{Fo} \leq$ $100 \%$; elle est fréquente si $60<\mathrm{Fo} \leq 79 \%$ ), elle est assez fréquente si $40<$ Fo $\leq 59 \%$, elle est espèce accessoire si $20<\mathrm{Fo} \leq 39 \%$ et elle est accidentelle si $\leq 20 \%$.

\section{$4 \quad$ RESULTATS}

4.1 Variations globales

4.1.1 Composition spécifique globale : Les inventaires réalisés de février 2016 à janvier 2017 ont permis d'identifier 63 espèces d'oiseaux (Figure 2) appartenant à 32 familles
3.3 Analyses statistiques: L'analyse de variances a servi à comparer les effectifs moyens saisonniers des espèces et les catégories d'espèces d'oiseaux. A la suite de cette comparaison, le test de comparaison et de classification de Newman-Keuls a été utilisé pour classer les espèces. Le Modèle Linéaire Généralisé (GLM) a été utilisé pour tester l'effet de la saison sur la distribution des oiseaux. Tous les tests statistiques ont été effectués avec le logiciel Statistica (Version 7.1).

et regroupées en 14 ordres. Globalement, l'ordre des Passériformes et la famille des Ardeidae sont les plus importants avec respectivement 12 familles $(46,87 \%)$ et sept espèces $(11,11 \%)$ (Tableau 1).

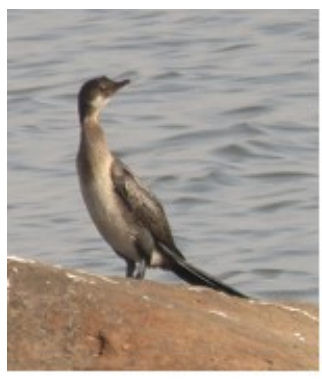

Microcarbo africans

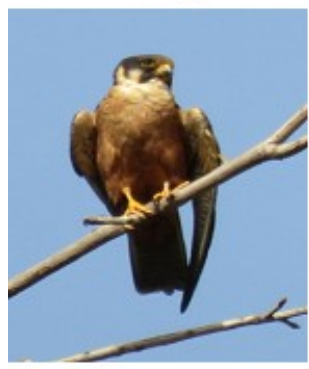

Falco cuvierii

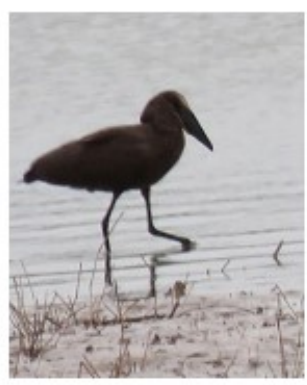

Scopus unbretta

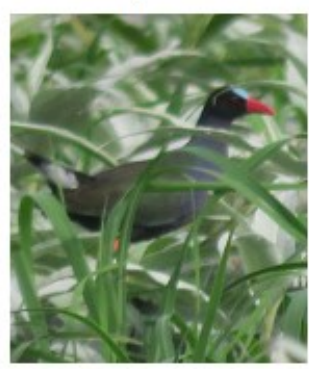

Porphyrio alleni

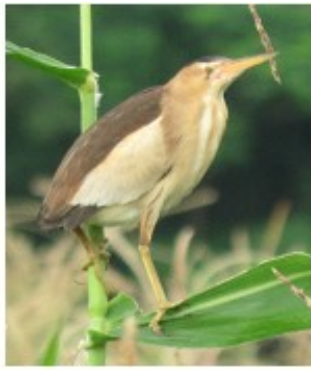

Ixobrychus minutus

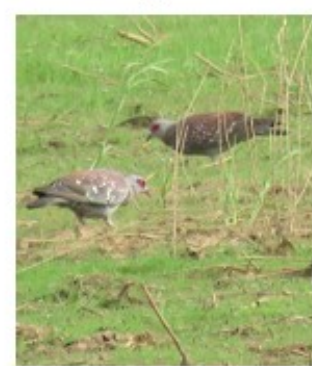

Columba guinea

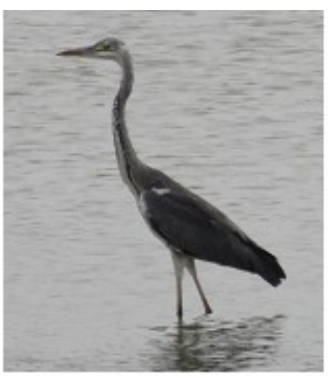

Ardea cinerea

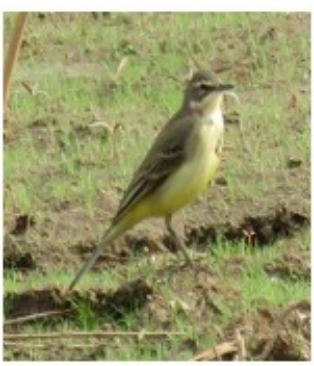

Motacilla flava

Figure 2 : Photographies de quelques espèces d'oiseaux observées au barrage urbain de Koko à Korhogo de février 2016 à janvier 2017

4.1.2 Structure globale : Le peuplement est dominé par les espèces résidentes avec 37 espèces (59 \%). Les espèces migratrices du paléarctique avec 12 espèces et les migrateurs intra-africains avec 16 espèces représentent respectivement $19,05 \%$ et $25,4 \%$ de l'effectif global. 
Tableau 1 : Bilan saisonnier des espèces observées au barrage urbain de Koko à Korhogo de février 2016 à janvier 2017

\begin{tabular}{|c|c|c|c|c|c|c|}
\hline \multirow[b]{2}{*}{ Ordres /Familles /Espèces } & \multicolumn{2}{|c|}{$\begin{array}{l}\text { Nombre } \\
\text { d'espèces }\end{array}$} & \multicolumn{2}{|c|}{$\begin{array}{c}\text { Statut } \\
\text { biogéographique }\end{array}$} & \multicolumn{2}{|c|}{$\begin{array}{c}\text { Fréquences } \\
\text { d'occurrence }(\%)\end{array}$} \\
\hline & SS & SP & SS & SP & SS (\%) & SP (\%) \\
\hline $\begin{array}{l}\text { SULIFORMES } \\
\text { Phalacrocoracidae } \\
\text { Microcarbo africanus (Gmelin, 1789) }\end{array}$ & + & + & $\mathbf{R}$ & $\mathbf{R}$ & Assez-fréquente $(40 \%)$ & Assez-fréquente $(42,85 \%)$ \\
\hline $\begin{array}{l}\text { PELECANIFORMES } \\
\text { Scopidae } \\
\text { Scopus umbretta Gmelin, 1789 } \\
\text { Ardeidae } \\
\text { Ixobrychus minutus (Linné, 1766) } \\
\text { Ardeola ralloides (Scopoli, 1769) } \\
\text { Bubulcus ibis (Linné, 1758) } \\
\text { Butorides striata (Linné, 1758) } \\
\text { Egretta garzetta (Linné, 1766) } \\
\text { Ardea purpurea Linné, 1766 } \\
\text { Ardea cinerea Linné, 1758 }\end{array}$ & $\begin{array}{l}+ \\
+ \\
+ \\
+ \\
+\end{array}$ & $\begin{array}{l}+ \\
+ \\
+ \\
+ \\
+ \\
+\end{array}$ & $\begin{array}{c}\mathrm{R} / \mathrm{P} / \mathbf{O} \\
\mathrm{R} / \mathrm{M} \\
\mathrm{R} \\
\mathrm{R} / \mathrm{M} / \mathbf{P} \\
\mathrm{R} / \mathbf{P} / \mathbf{O}\end{array}$ & $\begin{array}{c}\mathbf{R} \\
\mathbf{R} / \mathbf{P} / \mathbf{O} \\
\mathbf{R} / \mathbf{P} / \mathbf{O} \\
\mathbf{R} / \mathbf{M} \\
\mathbf{R} \\
\mathbf{R} / \mathbf{M} / \mathbf{P} \\
\mathbf{R} / \mathbf{P}\end{array}$ & $\begin{array}{c}\text { Très fréquente }(80 \%) \\
\text { Très fréquente }(80 \%) \\
\text { Fréquente }(60 \%) \\
\text { Très fréquente }(100 \%) \\
\text { Assez fréquente }(40 \%)\end{array}$ & $\begin{array}{l}\text { Assez-fréquente }(42,85 \%) \\
\text { Accessoire }(28,57 \%) \\
\text { Accidentelle }(14,28 \%) \\
\text { Assez-fréquente }(42,85 \%) \\
\text { Fréquente }(71,42 \%) \\
\text { Fréquente }(71,42 \%) \\
\text { Accessoire }(28,57 \%)\end{array}$ \\
\hline $\begin{array}{l}\text { ACCIPITRIFORMES } \\
\text { Accipitridae } \\
\text { Elanus caeruleus (Desfontaines, 1789) } \\
\text { Milvus migrans (Boddaert, 1783) } \\
\text { Accipiter tachiro (Daudin, 1800) }\end{array}$ & $\begin{array}{l}+ \\
+\end{array}$ & $\begin{array}{l}+ \\
+\end{array}$ & $\begin{array}{c}\mathbf{M} / \mathbf{P} / \mathbf{R} \\
\mathbf{R}\end{array}$ & $\begin{array}{c}\mathbf{R} \\
\mathbf{M} / \mathbf{P} / \mathbf{R}\end{array}$ & $\begin{array}{l}\text { Très fréquente }(100 \%) \\
\text { Accessoire }(20 \%)\end{array}$ & $\begin{array}{l}\text { Accessoire }(28,57 \%) \\
\text { Fréquente }\left(71,42^{\circ} \%\right)\end{array}$ \\
\hline $\begin{array}{l}\text { FALCONIFORMES } \\
\text { Falconidae } \\
\text { Falco cuvierii A. Smith, } 1830\end{array}$ & + & & $\mathbf{R}$ & & Accessoire $(20 \%)$ & \\
\hline $\begin{array}{l}\text { GALLIFORMES } \\
\text { Phasianidae } \\
\text { Francolinus bicalcaratus (Linné, 1766) }\end{array}$ & & + & & $\mathbf{R}$ & & Accidentelle $(14,28 \%)$ \\
\hline $\begin{array}{l}\text { GRUIFORMES } \\
\text { Rallidae } \\
\text { Porphyrio alleni Thomson, } 1842 \\
\end{array}$ & & + & & $\mathbf{M} / \mathbf{R}$ & & Accidentelle $(14,28 \%)$ \\
\hline $\begin{array}{l}\text { CHARADRIIFORMES } \\
\text { Jacanidae } \\
\text { Actophilornis africanus (Gmelin, 1789) } \\
\text { Microparra capensis (A. Smith, 1839) } \\
\text { Charadriidae } \\
\text { Vanellus senegallus (Linné, 1766) } \\
\text { Vanellus spinosus (Linné, 1758) } \\
\text { Scolopacidae }\end{array}$ & $\begin{array}{l}+ \\
+ \\
+ \\
+\end{array}$ & + & $\begin{array}{c}\mathbf{R} \\
\mathbf{R} / \mathbf{M} \\
\mathbf{R} / \mathbf{M} \\
\mathbf{P} / \mathbf{O}\end{array}$ & $\mathbf{P} / \mathbf{O}$ & $\begin{array}{l}\text { Fréquente }(60 \%) \\
\text { Accessoire }(20 \%) \\
\text { Assez fréquente }(40 \%) \\
\text { Fréquente }(60 \%)\end{array}$ & Assez-fréquente $(42,85 \%)$ \\
\hline
\end{tabular}


Tringa ochropus Linné, 1758

Tringa glareola Linné, 1758

Actitis bypoleucos (Linné, 1758)

\section{Columbidae}

Columba guinea Linné, 1758

Streptopelia decipiens (Hartlaud Finsch, 1870)

Streptopelia semitorquata (Ruppell, 1837)

Streptopelia senegalensis (Linné, 1766)

MUSOPHAGIFORMES

Musophagidae

Crinifer piscator (Boddaert, 1783)

\section{CUCULIFORMES}

\section{Cuculidae}

Chrysococcyx klaas (Stephens, 1815)

Chrysococcyx caprius (Boddaert, 1783)

Centropus senegalensis (Linné, 1766)

\section{APODIFORMES}

\section{Apodidae}

Cypsiurus parvus (Lichtenstein, 1823) Apus affinis (J. E. Gray, 1830)

\section{CORACIIFORMES}

\section{Alcedinidae}

Alcedo cristata Pallas, 1764

Halcyon senegalensis (Linné, 1766)

\section{Coraciidae}

Coracias naevius Daudin, 1800

\section{BUCEROTIFORMES}

\section{Bucerotidae}

Tockus fasciatus (Shaw, 1811)

Tockus nasutus (Linné, 1766)

\section{PASSERIFORMES}

\section{Hirundinidae}

Hirundo lucida Hartlaub, 1858

Motacillidae

Anthus leucophrys Vieillot, 1818

Motacilla flava Linné, 1758

\section{Pycnonotidae}

Pycnonotus barbatus (Desfontaines, 1789)

Atimastillas flavicollis (Swainson, 1837)

Thescelocicbla leucopleura (Cassin, 1855)

\begin{tabular}{|c|c|c|c|c|c|c|}
\hline & $\begin{array}{l}+ \\
+ \\
+\end{array}$ & + & $\begin{array}{l}\mathbf{P} \\
\mathbf{P} \\
\mathbf{P}\end{array}$ & $\mathbf{P}$ & $\begin{array}{l}\text { Assez fréquente }(40 \%) \\
\text { Assez fréquente }(40 \%) \\
\text { Assez fréquente }(40 \%)\end{array}$ & Accessoire $(28,57 \%)$ \\
\hline $\begin{array}{l}\text { sch, 1870) } \\
837) \\
\end{array}$ & $\begin{array}{l}+ \\
+ \\
+ \\
+\end{array}$ & $\begin{array}{l}+ \\
+ \\
+ \\
+\end{array}$ & $\begin{array}{c}\mathbf{R} \\
\mathbf{R} / \mathbf{M} \\
\mathbf{R} \\
\mathbf{R}\end{array}$ & $\begin{array}{c}\mathbf{R} \\
\mathbf{R} / \mathbf{M} \\
\mathbf{R} \\
\mathbf{R}\end{array}$ & $\begin{array}{l}\text { Très fréquente }(100 \%) \\
\text { Assez fréquente }(40 \%) \\
\text { Très fréquente }(80 \%) \\
\text { Très fréquente }(100 \%)\end{array}$ & $\begin{array}{c}\text { Très fréquente }(85,71 \%) \\
\text { Assez-fréquente }(42,85 \%) \\
\text { Très fréquente }(85,71 \%) \\
\text { Très fréquente }(100 \%)\end{array}$ \\
\hline & & + & & $\mathbf{R}$ & & Accidentelle $(14,28 \%)$ \\
\hline 3) & + & $\begin{array}{l}+ \\
+ \\
+\end{array}$ & $\mathbf{R}$ & $\begin{array}{c}\mathbf{M} / \mathbf{R} \\
\mathbf{M} / \mathbf{R} \\
\mathbf{R}\end{array}$ & Fréquente $(60 \%)$ & $\begin{array}{c}\text { Accidentelle }(14,28 \%) \\
\text { Assez-fréquente }(42,85 \%) \\
\text { Assez fréquente }(57,14 \%)\end{array}$ \\
\hline 3) & $\begin{array}{l}+ \\
+ \\
\end{array}$ & $\begin{array}{l}+ \\
+\end{array}$ & $\begin{array}{l}\mathbf{R} \\
\mathbf{R}\end{array}$ & $\begin{array}{l}\mathbf{R} \\
\mathbf{R}\end{array}$ & $\begin{array}{l}\text { Accessoire }(20 \%) \\
\text { Fréquente }(60 \%)\end{array}$ & $\begin{array}{c}\text { Assez-fréquente }(42,85 \%) \\
\text { Très fréquente }(100 \%)\end{array}$ \\
\hline & + & $\begin{array}{l}+ \\
+ \\
+ \\
+\end{array}$ & $\mathbf{R} / \mathbf{M}$ & $\begin{array}{l}\mathrm{R} / \mathrm{M} \\
\mathrm{M} / \mathrm{R} \\
\mathrm{R} / \mathrm{M}\end{array}$ & Très fréquente $(100 \%)$ & $\begin{array}{c}\text { Fréquente }(71,42 \%) \\
\text { Assez fréquente }(57,14 \%) \\
\text { Accidentelle }(14,28 \%)\end{array}$ \\
\hline & $\begin{array}{l}+ \\
+\end{array}$ & $\begin{array}{l}+ \\
+ \\
\end{array}$ & $\begin{array}{c}\mathbf{R} \\
\mathbf{M} / \mathbf{R}\end{array}$ & $\underset{\mathbf{M} / \mathbf{R}}{\mathbf{R}}$ & $\begin{array}{l}\text { Accessoire }(20 \%) \\
\text { Fréquente }(60 \%)\end{array}$ & $\begin{array}{c}\text { Accessoire }(28,57 \%) \\
\text { Assez-fréquente }(42,85 \%)\end{array}$ \\
\hline & $\begin{array}{l}+ \\
+ \\
+\end{array}$ & $\begin{array}{l}+ \\
+\end{array}$ & $\begin{array}{c}\mathbf{R} / \mathbf{M} \\
\mathbf{R} \\
\mathbf{P}\end{array}$ & $\begin{array}{c}\mathbf{R} / \mathbf{M} \\
\mathbf{R} \\
\mathbf{P}\end{array}$ & $\begin{array}{l}\text { Fréquente }(60 \%) \\
\text { Accessoire }(20 \%) \\
\text { Accessoire }(20 \%)\end{array}$ & $\begin{array}{c}\text { Très fréquente }(85,71 \%) \\
\text { Accessoire }(28,57 \%) \\
\text { Accidentelle }(14,28 \%)\end{array}$ \\
\hline 1789) & $\begin{array}{l}+ \\
+ \\
+\end{array}$ & + & $\begin{array}{l}\mathbf{R} \\
\mathbf{R} \\
\mathbf{R}\end{array}$ & $\mathbf{R}$ & $\begin{array}{l}\text { Très fréquente }(80 \%) \\
\text { Accessoire }(20 \%) \\
\text { Accessoire }(20 \%)\end{array}$ & Fréquente $(71,42 \%)$ \\
\hline
\end{tabular}




\section{Turdidae}

Turdus pelios Bonaparte, 1850

Muscicapidae

Cossypha albicapillus (Vieillot, 1818)

Sylviidae

Sylvia communis Latham, 1787

\section{Cisticolidae}

Hypergerus atriceps (Lesson, 1831)

Cisticola galactotes (Temminck, 1821)

Nectariniidae

Chalcomitra senegalensis (Linné, 1766)

\section{Laniidae}

Corvinella corvina (Shaw, 1809)

Malaconotidae

Laniarius barbarus (Linné, 1766)

Tchagra senegalus (Linné, 1766)

\section{Corvidae}

Ptilostomus afer (Linné, 1766)

Corvus albus Müller, 1776

\section{Passeridae}

Passer griseus (Vieillot, 1817)

\section{Ploceidae}

Ploceus cucullatus (Müller, 1776)

Malimbus scutatus (Cassin, 1849)

Euplectes franciscanus (Isert, 1789)

Euplectes afer (J. F. Gmelin, 1789)

\section{Estrildidae}

Uraeginthus bengalus (Linné, 1766)

Lagonosticta senegala (Linnaeus, 1766)

Lonchura cucullata (Swainson, 1837)

Fringillidae

Crithagra mozambica (Statius Muller, 1776)

\begin{tabular}{|c|c|c|c|}
\hline & + & & $\mathbf{R}$ \\
\hline+ & & $\mathbf{R}$ & \\
\hline+ & + & $\mathbf{P}$ & $\mathbf{P}$ \\
\hline+ & + & $\mathbf{R}$ & $\mathbf{R}$ \\
\hline+ & + & $\mathbf{R}$ & $\mathbf{R}$ \\
\hline+ & & $\mathbf{R} / \mathbf{M}$ & \\
\hline+ & + & $\mathbf{R}$ & $\mathbf{R}$ \\
\hline+ & + & $\mathbf{R}$ & $\mathbf{R}$ \\
\hline+ & + & $\mathbf{R}$ & $\mathbf{R}$ \\
\hline+ & + & $\mathbf{R}$ & $\mathbf{R}$ \\
\hline+ & + & $\mathbf{R}$ & $\mathbf{R}$ \\
\hline+ & + & $\mathbf{R}$ & $\mathbf{R}$ \\
\hline+ & + & $\mathbf{R}$ & $\mathbf{R}$ \\
\hline & + & & $\mathbf{R}$ \\
\hline+ & + & $\mathbf{M}$ & $\mathbf{M}$ \\
\hline+ & + & $\mathbf{R}$ & $\mathbf{R}$ \\
\hline+ & + & $\mathbf{R}$ & $\mathbf{R}$ \\
\hline+ & + & $\mathbf{R}$ & $\mathbf{R}$ \\
\hline+ & + & $\mathbf{R}$ & $\mathbf{R}$ \\
\hline & + & & $\mathbf{R}$ \\
\hline 4 & 52 & & \\
\hline
\end{tabular}

Accessoire (20\%)

Accessoire (20\%)

Accessoire (20\%)

Très fréquente $(80 \%)$

Accessoire (20\%)

Assez fréquente (40\%)

Assez fréquente $(40 \%)$

Accessoire $(20 \%)$

Assez fréquente $(40 \%)$ Assez fréquente $(40 \%)$

Fréquente $(60 \%)$

Très fréquente $(100 \%)$

Assez fréquente $(40 \%)$ Accessoire $(20 \%)$

Assez fréquente $(40 \%)$ Accessoire $(20 \%)$

Très fréquente $(100 \%)$
Assez-fréquente (42,85\%)

Fréquente $(71,42 \%)$

Accessoire (28,57\%)

Accessoire (28,57\%)

Assez-fréquente (42,85\%)

Fréquente $(71,42 \%)$

Accidentelle (14,28\%)

Assez-fréquente (42,85\%)

Assez-fréquente $(42,85 \%)$

Assez fréquente $(57,14 \%)$

Très fréquente $(85,71 \%)$

Accidentelle (14,28\%)

Fréquente $(71,42 \%)$

Assez fréquente $(57,14 \%)$

Très fréquente $(85,71 \%)$

Accessoire (28,57\%)

Très fréquente $(100 \%)$

Accidentelle (14,28\%)

Total

(+ : espèce présente ; SS : saison sèche, SP : saison pluvieuse ; R : Résident ; P : Migrateur du Paléarctique ; M : Migrateur intra-africain ; O :

Occasionnel) 
Vingt espèces ont un statut mixte (résidentemigratrice-occasionnelle) et représentent $32 \%$ du peuplement (Tableau 1). La caractérisation des espèces d'oiseaux sur la base de la fréquence d'occurrence révèle que les espèces accidentelles, avec 23 espèces, sont dominantes dans le peuplement $(36,51 \%)$. Les espèces assez fréquentes, les espèces accessoires et les espèces très fréquentes $(\mathrm{N}=9-16)$ sont moyennement représentées $(14,28 \%$ à $25,4 \%)$ alors que les espèces fréquentes $(\mathrm{N}=5)$ sont faiblement représentées (7,94\%) (Tableau 1).

\subsection{Variations saisonnières}

4.2.1 Composition spécifique saisonnière : Sur un total de 14 ordres, 11 ordres sont présents en saison sèche $(78,57 \%)$ alors que 12 ordres ont été observés en saison des pluies $(85,71 \%)$. Les ordres spécifiquement observés au cours des différentes saisons sont les Falconiformes en saison sèche; les Musophagiformes, les Gruiformes et les Galliformes en saison des pluies (Figure 3). L'ordre des Passériformes est le mieux représenté quelle que soit la saison. Les ordres secondairement représentés sont les charadriiformes. Les autres ordres ont été faiblement représentés au cours des différentes saisons (Figure 3). Le Modèle Linéaire Généralisé confirme cette observation en relevant que la distribution des ordres d'oiseaux a varié de manière significative avec la saison $(\mathrm{ddl}=1 ; \mathrm{W}=7,1 ; \mathrm{p}<0,05)$.

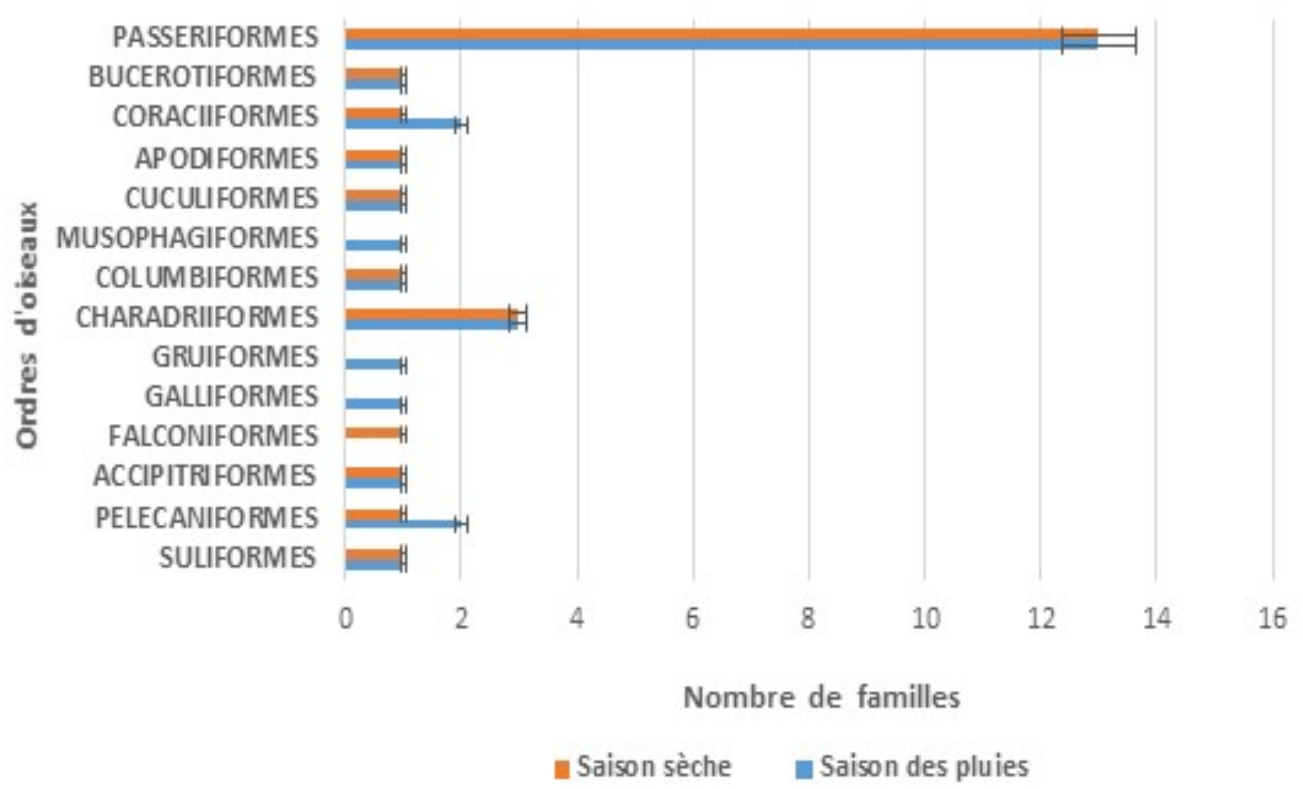

Figure 3 : Variations saisonnières des ordres d'oiseaux du barrage de Koko à Korhogo de février 2016 à janvier 2017

En saison sèche, 25 familles ont été observées $(71,42 \%)$ alors que 29 familles ont été enregistrées en saison des pluies (90,62\%). Les familles uniquement observées en saison sèche ont été les Nectariniidae, les Muscicapidae et les Falconidae. Celles uniquement observées en saison des pluies sont les Fringilidae, les Turdidae, les Coraciidae, les Musophagidae, les Rallidae, les Phasianidae et les Scopidae (Figure 4). La famille la mieux représentée est celle des
Ardeidae, en toute saison. Cette famille est suivie des familles des Columbidae et des Ploceidae. Le reste des familles est faiblement représenté quelle que soit la saison (Figure 4). Le Modèle Linéaire Généralisé confirme cette observation en relevant que la distribution des familles d'oiseaux a varié de manière hautement significative avec la saison (GLM : $\mathrm{ddl}=1$; $\mathrm{w}=10,87 ; \mathrm{p}<0,001)$. 


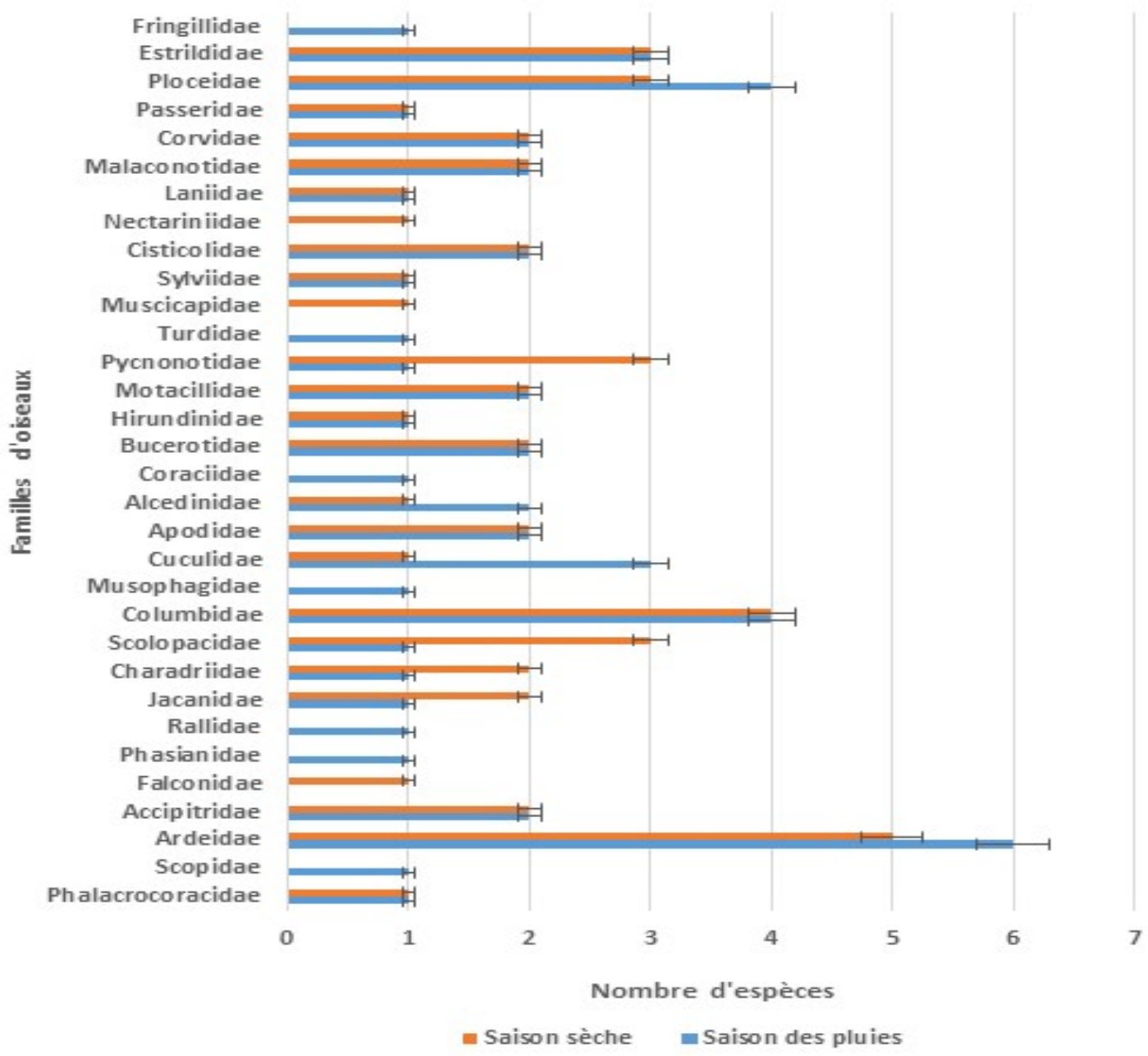

Figure 4: Variations saisonnières des familles d'oiseaux du barrage de Koko à Korhogo de février 2016 à janvier 2017

\subsubsection{Distribution saisonnière des communautés : Les espèces les plus} fréquentes en saison sèche sont au nombre de sept. Il s'agit de Aigretta garzetta, de Milvus migrans, de Columba guinea, de Streptopelia senegalensis, de Alcedo cristata, de Ploceus cucullatus et de Lonchura cucullata. En saison des pluies, trois espèces sont les plus fréquentes. Ce sont Lonchura cucullata, Apus affinis et Streptopelia senegalensis. La comparaison globale saisonnière du nombre d'espèces montre que le plus grand nombre d'espèces a été observé en saison des pluies $(\mathrm{N}=52$, soit $82,54 \%$ des espèces totales) comparé à la saison sèche $(\mathrm{N}=49$, soit $77,78 \%$ des espèces totales) (Tableau 1). La moyenne des espèces en saison sèche de $24 \pm$
4,43 a oscillé entre un minimum de 17 espèces (décembre) et un maximum de 35 espèces (mars). En saison des pluies, la moyenne de 25 $\pm 1,27$ espèce observée a variée entre un minimum de 23 espèces (mai) et un maximum de 29 espèces (août) (Tableau 1). L'analyse de variances ne révèle aucune différence significative entre les nombres moyens saisonniers d'espèces d'oiseaux $(\mathrm{ddl}=1$; $\mathrm{F}=0,12 ; \mathrm{p}>0,05)$.

4.2.3 Structure saisonnière : La distribution des statuts biogéographique des espèces d'oiseaux respectivement en saison sèche ( $\mathrm{ddl}=7 ; \mathrm{F}=172,7 ; \mathrm{p}<0,0001)$ et en saison des pluies ( $\mathrm{ddl}=7 ; \mathrm{F}=149,75 ; \mathrm{p}<0,0001)$ a varié de manière hautement significative. Le test 
Post-Hoc de Newman-Keuls révèle que les espèces résidentes sont les plus abondantes en toute saison. Les espèces à double origine en l'occurrence les espèces résidentes et migratrices intra-africain $(\mathrm{R} / \mathrm{M})$ sont moyennement représentées alors que les espèces à autres statuts sont faiblement présentes en toute saison (Figure 5). En effet, le Modèle Linéaire Généralisé confirme que la distribution des espèces selon leur statut biogéographique a varié de manière hautement significative avec la saison $(\mathrm{ddl}=1 ; \mathrm{W}=9,51$; $\mathrm{p}<0,001)$.

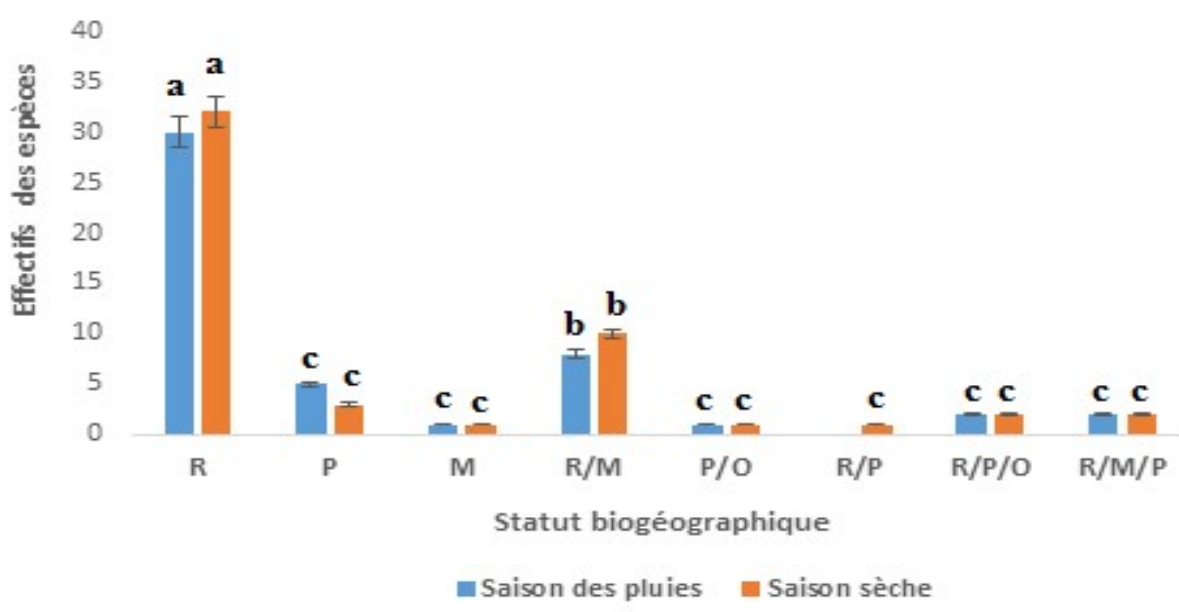

Figure 5: Variations saisonnières des statuts biogéographiques des espèces d'oiseaux du barrage de Koko à Korhogo de février 2016 à janvier 2017 (R : Résident ; P : Migrateur du Paléarctique ; M : Migrateur intra-africain ; $\mathrm{O}$ : Occasionnel)

La distribution des catégories d'espèces d'oiseaux sur la base des fréquences respectivement en saison sèche $(\mathrm{ddl}=4 ; \mathrm{F}=$ $35,81 ; \mathrm{p}<0,0001)$ et en saison des pluies (ddl $=4 ; \mathrm{F}=7,64 ; \mathrm{p}<0,001)$ a varié de manière hautement significative. En saison sèche, le test Post-Hoc de comparaison et de classification de Newman-Keuls révèle que les espèces accessoires, assez-fréquentes et très fréquentes ont été les plus abondantes. Les espèces fréquentes sont moyennement représentées alors que les espèces accessoires sont faiblement présentes (Figure 6). Pendant la saison des pluies, les espèces assez-fréquentes ont été les plus nombreuses tandis que les autres catégories d'espèces sont peu représentées (Figure 6). En effet, le Modèle Linéaire Généralisé confirme cette observation en relevant que la distribution des catégories d'espèces d'oiseaux a varié de manière hautement significative avec la saison $(\mathrm{ddl}=1$; $\mathrm{W}=21,12 ; \mathrm{p}<0,0001)$. 


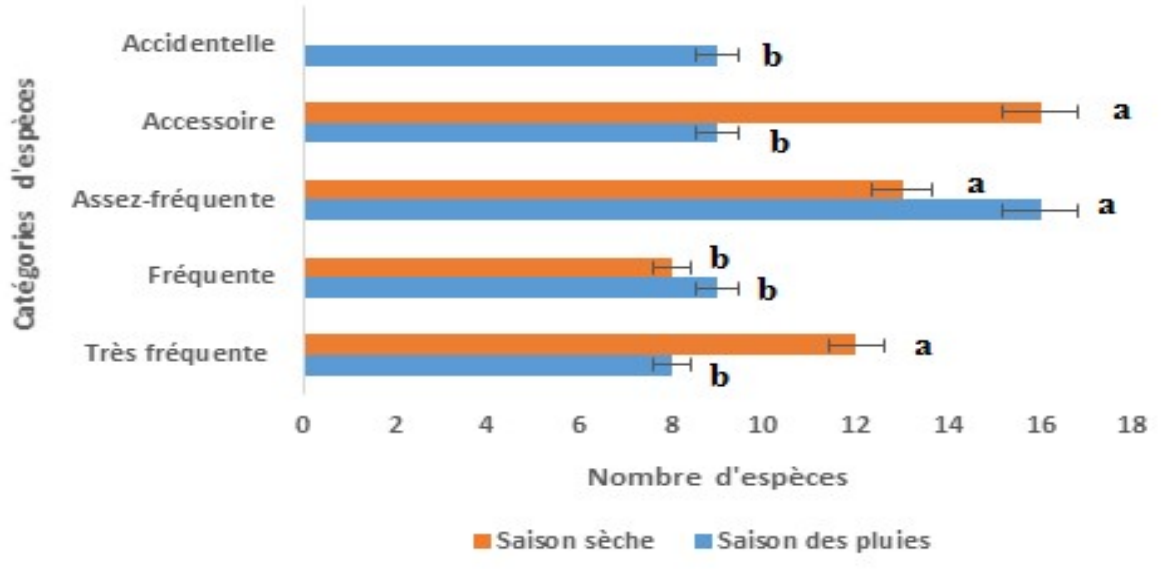

Figure 6: Variations saisonnières des catégories d'espèces d'oiseaux du barrage de Koko à Korhogo de février 2016 à janvier 2017

\section{DISCUSSION}

Les inventaires qualitatifs de l'avifaune du barrage urbain de Koko de Korhogo ont permis d'identifier 63 espèces d'oiseaux. Ce nombre est inférieur aux 138 espèces, 218 espèces et 165 espèces inventoriées dans la zone humide de Grand-Bassam (YaokokoréBéibro, 2010 ; Odoukpé et al., 2014 ; Yaokokoré-Béibro et al., 2015 b) ainsi qu'aux 166 espèces observées sur les lacs urbain de Yamoussoukro (Konan et al., 2015). Nos résultats sont proches des 73 espèces d'oiseaux déterminées dans la zone marécageuse de la Djibi à Abidjan (Yaokokoré-Béibro et al., 2015 a) mais sont supérieurs aux 29 espèces identifiées dans la zone humide du Kashmir en Inde (Ahmad et Bhat, 2017). Ces différences pourraient être liées d'une part à l'étendue des zones d'études pour les travaux menés en Côte d'Ivoire, et, d'autre part, à la diversité, à l'abondance des ressources alimentaires et à l'hétérogénéité des habitats offerts par les écosystèmes (Yaokokoré-Béibro et al., 2015 a, b). Les écarts observés avec ceux du Kashmir se rattacheraient à la période d'étude relativement brève, qui a été de six mois. Les résultats ont relevé que l'ordre des Passériformes est le mieux représenté à l'instar des études effectuées sur les lacs urbain de la ville de Yamoussoukro (Konan et al., 2015) et de la zone marécageuse de la Djibi à Abidjan
(Yaokokoré-Béibro et al., 2015 a). Cependant, des études ont montré que chez les oiseaux d'eau, les non-passériformes ont constitué le groupe le plus important dans la zone humide de Grand-Bassam en Côte d'Ivoire (Yaokokoré-Béibro et al., 2010 ; Odoukpé et al., 2014 ; Yaokokoré-Béibro et al., 2015 b) et dans la zone humide du Kashmir en Inde (Ahmad et Bhat, 2017).

La famille des Ardeidae a été la plus riche en espèces. Nos résultats sont similaires aux travaux menés en Côte d'Ivoire dans la zone humide de Grand-Bassam, dans la zone marécageuse de la Djibi à Abidjan et sur les lacs urbains de la ville de Yamoussoukro (Yaokokoré-Béibro et al., 2010; Konan et al., 2015; Yaokokoré-Béibro et al., 2015 b). En outre, Odoukpé et al. (2014) ont identifié en plus les familles des Accipitridae et des Scolopacidae comme étant les plus diversifiées tandis qu'en Inde, dans la zone humide du Kashmir, ce sont les Alcedinidae qui sont les plus diversifiées (Ahmad et Bhat, 2017).

La plus grande richesse spécifique des oiseaux a été observée au cours de la saison pluvieuse $(\mathrm{N}$ $=52$ espèces). Nos résultats sont contraires aux travaux de Konan et al. (2015) sur les lacs urbain de la ville de Yamoussoukro, pour qui, le plus grand nombre d'espèces a été identifié en saison sèche. Cette différence serait liée à la 
diversité d'habitats. En effet, les pluies ont pour corollaire la remontée du niveau de l'eau et de la repousse de la végétation, qui permettent de distinguer différents milieux (eau, berge, prairie et savane arborée), donc de niches écologiques, ainsi que des ressources alimentaires variées, qui attireraient un grand nombre d'espèces d'oiseaux (Castano-Villa et al., 2014). Par ailleurs, certains de ces environnements pourraient servir de perchoir, de dortoir, de site de reproduction, de site de nidification et de refuge contre d'éventuels prédateurs ou en cas de dérangement (Lougbegnon et al., 2010; Khaffou et al., 2013; Konan et al., 2015; Yaokokoré-Béibro et al., 2015 a, b ; Nasasagare et al., 2017 ; Castano-Villa et al., 2019). Les distributions saisonnières qualitatives des oiseaux ont relevé que les ordres et les familles d'oiseaux sont repartis selon des saisons spécifiques. Ce fait pourrait s'expliquer par leur spécialisation en relation avec leurs différentes activités bioécologiques et leur sécurité (Konan et al., 2015 ; Nasasagare et al., 2017 ; CastanoVilla et al., 2014 ; Castano-Villa et al., 2019). Du point de vue de leur statut biogéographique, la structure globale du peuplement observé, dominée par les espèces résidentes est semblable à celles des études réalisées dans la zone marécageuse de la Djibi (YaokokoréBéibro et al., 2015 a), dans une zone de riziculture dans la zone humide de GrandBassam (Odoukpé et al., 2014) et dans la zone humide d'importance internationale de Grand-

\section{CONCLUSION}

L'étude de l'avifaune du barrage urbain de Koko à Korhogo a permis d'inventorier 63 espèces, qui se répartissent en 32 familles et 14 ordres. Globalement, l'ordre des Passériformes et la famille des Ardeidae ont été les mieux représentés. Les espèces résidentes et la catégorie d'espèces accidentelles ont été les plus abondantes. Le plus grand nombre d'espèces d'oiseaux a été observé pendant la saison des pluies. Les ordres des Musophagiformes, des Coraciiformes et des Cuculiformes sont caractéristiques de la saison pluvieuse alors les ordres des Suliformes, des Passeriformes, des
Bassam (Yaokokoré-Béibro et al., 2010 ; Yaokokoré-Béibro et al., 2015 b). La dominance des espèces résidentes est maintenue également au cours des différentes saisons. Cette observation pourrait être due au fait que le milieu offre en toute saison des conditions idéales à l'installation et au maintien des espèces résidentes. Par ailleurs, ce barrage urbain n'offre pas de plage vasière susceptible d'attirer les migrateurs du paléarctique.

La fréquence d'occurrence a relevé au niveau global que les espèces accidentelles ont été les plus abondantes dans le peuplement. Cette présence pourrait s'expliquer par le fait que ces espèces exploiteraient de façon ponctuelle ce milieu en vue de tirer profit des conditions offertes par cette zone humides (Castano-Villa et al., 2014 ; Ahmad et Bhat, 2017). En saison des pluies, les espèces assez-fréquentes ont été les plus nombreuses alors que les catégories d'espèces accessoires et très fréquentes l'ont été en saison sèche. Ces différences pourraient s'expliquer par les conditions particulières d'habitat, de reproduction et de disponibilité de ressources alimentaires offertes par le milieu au cours des différentes saisons. Les saisons et les conditions du milieu interfèreraient afin de créer les conditions de présence propres aux différentes catégories d'espèces d'oiseaux (Castano-Villa et al., 2014; Konan et Yaokokoré-Béibro, 2015; Ahmad et Bhat, 2017).

Charadriiformes, des Buceriformes et des Columbiformes sont représentatifs de la saison sèche. En toute saison, les espèces résidentes ont été les plus nombreuses. Les espèces assezfréquentes ont été les plus abondantes en saison des pluies tandis que les espèces accessoires, assez-fréquentes et très fréquentes ont été les plus représentées en saison sèche. La saison a influencé la distribution des ordres, des familles et des espèces d'oiseaux. Ce site urbain est soumis à diverses pressions anthropiques liées à la pratique de l'agriculture (maraîchers) en amont, autour du barrage. Cette activité 
présenterait des risques pour la survie de cette communauté d'oiseaux du fait de l'utilisation d'engrais et de pesticides, qui sont entrainés par les eaux de pluies dans le barrage. Cela a pour

\section{$7 \quad$ REFERENCES}

Ahmad MA. and Bhat IA : 2017. Bird abundance of a flood plain wetland of Kashmir Himalayas. International Journal of Zoology Studies 2:10-13.

Bibby CJ, Burgess ND. and Hill DA: 1992. Bird Census Techniques. Academic Press, London, England. 257 pp.

Borrow N. and Demey R: 2001. Birds of western Africa. Christopher Helm, London, England.832 pp.

Borrow N. et Demey R: 2008. Guide des Oiseaux d'Afrique de l'Ouest. Delachaux et Niestlé, Paris, France. 508 pp.

Bryce SA, Hughes RM. and Kaufmann PR: 2002. Development of a bird integrity index: using bird assemblages as indicators of riparian condition. Environmental Management 30:294-310.

Brooks T, Andriamaro L, Gereau R, Hawkins F, Howell II PP, Luke Q, Matiku P, McKnight MW, Msuya C, Mugo R, Rabarison H, Rakotobe ZL. and Randrianasolo H: 2007. Objectifs et priorités pour la conservation des Oiseaux et de la biodiversité d'Afrique. Ostrich 78: 115-234.

Castano-Villa GJ, Ramos-Valencia SA. and Fontúrbel FE : 2014. Fine-scale habitat structure complexity determines insectivorous bird diversity in a tropical forest. Acta Oecologica 61:19-23.

Castaño-Villa GJ, Santisteban-Arenas R, Hoyos-Jaramillo A, Estévez-Varon JV. and Fonturbel FE: 2019. Foraging behavioural traits of tropical insectivorous birds lead to dissimilar communities in contrasting forest habitats. Wildlife Biology 1: 1-6.

Chappuis C: 2000. Oiseaux d'Afrique. 15 CDs. Paris, France. Société d'étude Ornithologique. répercussion la pollution et l'intoxication, qui constitueraient d'éventuelles menaces pour la reproduction des espèces d'oiseaux d'eau.

Conservation International : 2001. De la forêt à la mer: Les liens de la biodiversité de la Guinée au Togo. Conservation International, Washington, USA. 78 pp.

Coulibaly T: 2014. Diversité et dégât des termites dans les vergers de manguier de la région de Korhogo (Côte d'Ivoire) et essai de lutte par utilisation d'extrait aqueux de trois plantes locale. Thèse de doctorat d'Ecologie Tropicale, Université Felix Houphouët-Boigny, Abidjan, Côte d'Ivoire. 174 pp.

Djakou R. et Thanon SY: 1988. Ecologie Afrique intertropicale. Edition Bordas, Paris, France. 191 pp.

El Agbani MA. et Dakki M : 2005. Importance ornithologique du complexe des zones humides de la région de Smir. Travaux de l'Institut Scientifique 4: 61-64.

Khaffou M, Chalaoui A. and Samih M: 2013. Les habitats utilisés par le Tadorne casarca (Tadorna ferruginea) dans la zone humide d'Aguelmam Sidi Ali-Site Ramsar-Moyen Atlas- Maroc. International Journal of Biological and Chemical Sciences 7: 598-606.

Konan EM, Yaokokoré-Béibro KH, Odoukpé KSG et Kouadja KES : 2014. Avifaune de la ville de Yamoussoukro, centre de la Côte d'Ivoire. European Scientific Journal 10: 63-75.

Konan EM. et Yaokokoré-Béibro KH : 2015. Variation temporelle du peuplement aviaire des écosystèmes lacustres de la ville de Yamoussoukro, centre de la Côte d'Ivoire. International Journal of Biological and Chemical Sciences 9: 25662581.

Konan EM, Yaokokoré-Béibro KH. et Odoukpé KSG : 2015. Richesse spécifique et abondance des oiseaux des dix lacs urbains de la ville de 
Yamoussoukro, dans le centre de la Côte d'Ivoire. International Journal of Innovation and Applied Studies 10: 217-225.

Kouadio KP, Yaokokoré-Béibro KH, Odoukpé KSG, Konan EM. et Kouassi KP : 2014. Diversité avifaunique du Parc National du Banco, Sud-Est Côte d'Ivoire. European Journal of Scientific Research 125: 384-398.

Lougbegnon TO, Codjia JC. et Libois RM : 2010. Distribution de l'avifaune des milieux forestiers de substitution (plantation et jachères) au Sud du Bénin en relation avec les facteurs de l'habitat. International Journal of Biological and Chemical Sciences 4: 1191-1216.

Nasasagare RP, Ndayisaba ED. et Libois R: 2017. La déprédation non aléatoire chez les oiseaux granivores du marais de Kagogo-Gisumo au Burundi. Bulletin scientifique sur l'environnement et la biodiversité $2: 1-8$.

Odoukpé KSG, Yaokokoré-Béibro HK, Kouadio PK. et Konan ME: 2014. Dynamique du peuplement des Oiseaux d'une riziculture et ses environs dans la zone humide d'importance internationale de Grand-Bassam. Journal of Applied Biosciences 79: 6909-6925.

Subramanya S: 1994. Non-random foraging in certain bird pests of field crops. Journal of Biosciences 19: 369-380.

Williams M: 1993. Wetlands: A threatened landscape. Blackwell Publishers, Oxford, USA. 420 pp.

Yaokokoré-Béibro KH: 2001. Avifaune des forêts classées de l'Est de la Côte d'Ivoire : Données sur l'écologie des espèces et effet de la déforestation sur les peuplements. Cas des forêts classées de la Béki et de la Bossématié (Abengourou). Thèse de Doctorat, Université de Cocody, Côte d'Ivoire. 245pp.

Yaokokoré-Béibro KH : 2010. Oiseaux du Parc National des Iles Ehotilé, sud-est Côte d'Ivoire. Malimbus 32: 89-102.
Yaokokoré-Béibro HK, N'guessan MA, Odoukpé KSG, Zouzou EJ, N'douba V. et Kouassi PK: 2010. Premières données sur les oiseaux de la zone humide d'importance internationale de Grand-Bassam (Côte d'Ivoire). International Journal of Biological and Chemical Sciences 4: 2169-2180.

Yaokokoré-Béibro KH, Koné YS. et Odoukpé KSG : 2015 a. Avifaune d'un milieu marécageux urbain dans la commune de Cocody. International Journal of Innovation and Scientific Research 18: 99-108.

Yaokokoré-Béibro KH, Gueye MF, Koné YS. et Odoukpé KSG : 2015 b. Biodiversité urbaine des Oiseaux dans la Zone humide d'Importance Internationale de Grand-Bassam (Sud-Est de la Côte d'Ivoire). International Journal of Innovation and Applied Studies 11: 339-349.

Zean GM, Ahon DB. et Koffi BJC: 2018. Peuplement avifaunique du campus universitaire Jean Lorougnon Guédé, Daloa et sa périphérie (Centre-Ouest de la Côte d'Ivoire). International Journal of Biological and Chemical Science 12: 25032518. 